\title{
Auf dem Weg in eine neue Medizin
}

\section{Felicitas Witte}

Dr. med., Medizinjournalistin

Beim 11. Roundtable des Zurich Heart House diskutierten Experten aus Medizin, Industrie und Politik neue Lösungen in der Gesundheitspolitik. Ihr Fazit:

Die Medizin wird sich in den kommenden Jahren noch mehr ändern als bisher.

Zum elften Mal fand kürzlich der Roundtable der Stiftung für Herz- und Kreislaufforschung in Zürich (Zurich Heart House, ZHH) statt*. Thomas Lüscher, Präsident des ZHH und Direktor der Kardiologie am Unispital, wollte wieder Experten aus Medizin, Industrie und Politik zusammenbringen, um das Verständnis füreinander zu erhöhen und Lösungen zu finden. "Wir haben einen Roundtable», sagte Rita Ziegler, Vorsitzende der Spitaldirektion am Unispital Zürich. "Nun müssen wir schauen, dass das Ding auch rund wird.» Mit «dem Ding» kann Frau Ziegler vieles gemeint haben, was auf der Veranstaltung angesprochen wurde: die neuen Prioritäten der Schweizer Gesundheitspolitik, die Medizin im Wandel der Zeit, Health Technology Assessment, Spitalmarketing oder den neuen Pharmakodex - vermutlich bezog sie sich auf alle Themen.

Das Schweizer Gesundheitssystem scheint schon ziemlich rund, nur die Ecken müssten noch etwas geglättet werden. «Wir haben ein gutes Gesundheitssystem, das hören wir auch immer wieder aus dem Ausland», sagte Stefan Spycher, Vizedirektor des Bundesamtes für Gesundheit (BAG) in Bern. Aber wozu braucht es dann eine "Agenda 2020 »? «Vor allem weil es zu viele chronische Krankheiten gibt, und viele leiden nicht nur unter einer», fasste Spycher die Ergebnisse einer Untersuchung des Schweizerischen Gesundheitsobservatoriums zusammen [1]. Das verursacht enorme Kosten: Allein die Behandlung von Herz-Kreislauf-Krankheiten kostet pro Jahr mehr als 15 Milliarden Franken. Dabei seien die meisten Fakto- ren, die dazu führen, vermeidbar, sagte Spycher durch eine bessere Prävention, neu im BAG «Gesundheitsförderung und Krankheitsvorbeugung» genannt. eHealth soll in Zukunft eine entscheidende Rolle dabei spielen, wie sich die Versorgung verbessern lässt: So soll eHealth die Verschreibung von Medikamenten erleichtern, und die elektronische Patientenakte soll eine lückenlose Dokumentation ermöglichen und den Patienten besser betreuen helfen. «Unsere Agenda ist aber nicht in Stein gemeisselt», sagte Spycher. "Alle Partner können und müssen mitmachen, und wir brauchen Ihren Input.»

\section{Dr. Google wird den Arzt nicht ersetzen}

Urs Stoffel, Präsident der Ärztegesellschaft des Kantons Zürich, nahm das Publikum mit auf eine Reise in die «Cyber-Medizin». Schon jetzt gibt es Versuche in Japan mit Robotern, die Patienten waschen oder ihnen das Essen ans Bett bringen. "Alle drei Jahre verdoppelt sich das medizinische Wissen. Wir sind immer einige Schritte hintendran, wenn wir aktuell bleiben wollen", sagte Stoffel. Er warnte jedoch davor, sich zu sehr von der Technik einfangen zu lassen. «Wir sollten uns gut überlegen, wofür wir das Geld einsetzen.»

Als weiteren Punkt einer "neuen Medizin" nannte Stoffel die zunehmende Digitalisierung: Schon jetzt informieren sich viele Patienten über das Internet und suchen Rat bei «Dr. Google». Der könne aber nur sichten und nicht gewichten, so Stoffel. «Wir Ärzte www.zhh.ch/

carta-2014-programm

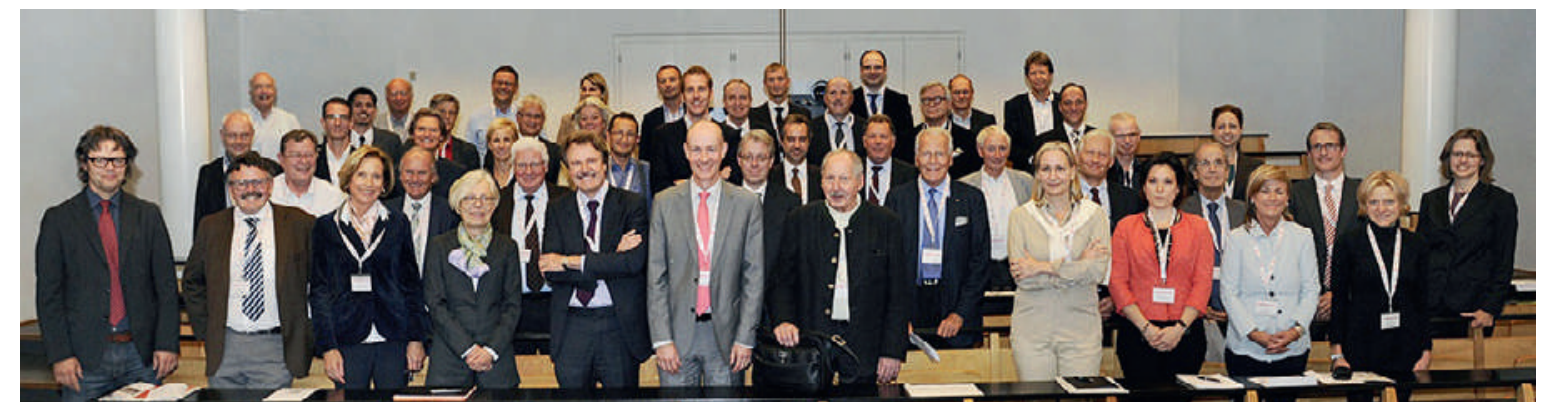

Lächeln für den Fotografen: die Teilnehmer des 11. Roundtable des Zurich Heart House. 


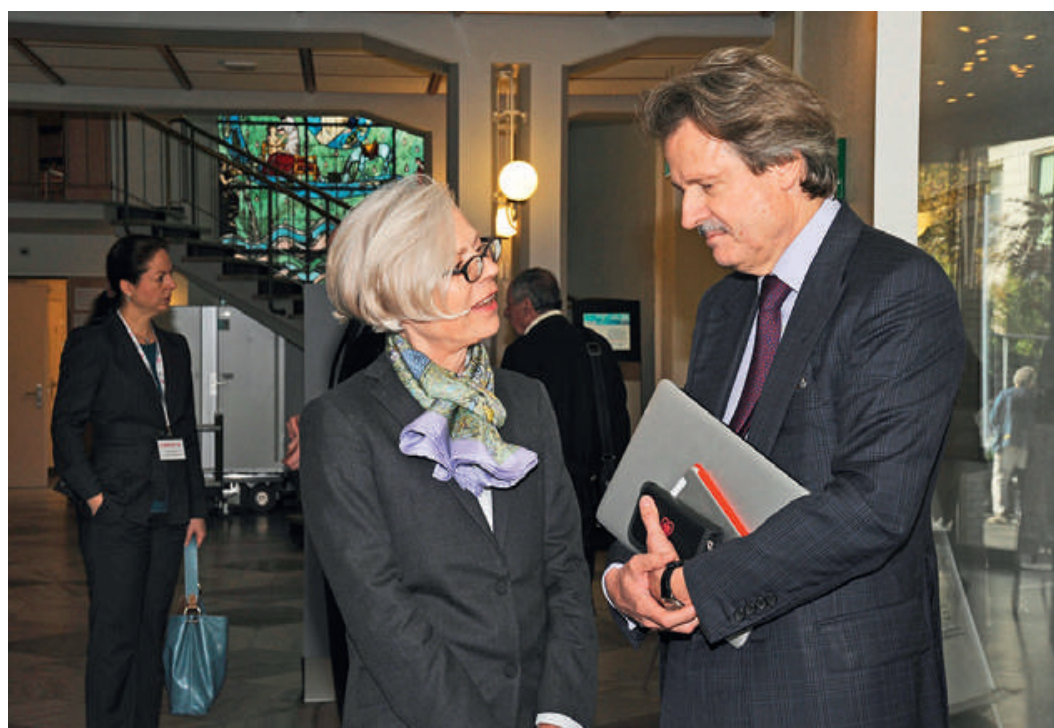

Thomas Lüscher hat den Roundtable organisiert. Rita Ziegler meint: «Nun müssen wir schauen, dass das Ding auch rund wird.»

werden weiterhin gebraucht, um die Daten zu interpretieren. Keine App, keine Internetseite könne Empathie und Vertrauen zum Arzt ersetzen.

Die Lösung sieht Stoffel in einem «multidisziplinären» Ansatz: Alle Versorgungsstrukturen müssen genutzt werden, die an die Bedürfnisse der Bewohner angepasst sind: Walk-in-Praxen, Permanence, Ärztefon, und auch der Hausarzt - aber nicht nur er alleine. Callcenter können den Patienten regelmässig anrufen und ihn mit seiner chronischen Krankheit betreuen. In Gegenden mit schlechter Versorgung könnten mobile Arztpraxen ähnlich wie der «MigrosWagen» von früher die Grundversorgung gewährleisten. Die digitalen Medien könnten die Behandlung von Patienten erleichtern, etwa der sogenannte Watson-Computer, der bei der Diagnose kniffeliger Fälle hilft, mobile Echo-Kardiographien, Dermatoskope oder ein mit dem iPhone kombinierter Diabetes-Kit.

\section{Ein neuer Pharmakodex}

Aus der "Cyber-» in die "reale Medizinwelt» zurück holte Lorenz Borer das Publikum. Borer ist bei Novartis zuständig für alles, was hilft, damit die Pharmafirma auf dem Markt erfolgreich ist. Borer berichtete über den neuen Pharma-Kooperations-Kodex (PKK), der ab 2015 gilt. Dieser sieht vor, dass die Firmen freiwillig geldwerte Leistungen von Pharmafirmen an Ärzte, Apotheker und Gesundheitsorganisationen wie Spitäler oder Ärzte-Netzwerke offenlegen. 50 Firmen haben den PKK schon unterschrieben. Als geldwerte Leistungen gelten zum Beispiel: Geld für klinische Studien, Zuwendungen für Fortbildungen, für Beratungs- und Dienstleistungen wie Advisory Boards felicitas.witte[at]icloud.com
Seefeldstrasse 285 oder Referate und für die Teilnahme an Fachveranstaltungen inklusive Fahrtkosten oder Unterkunft. Mindestens ein Drittel muss hierbei aber der Teilnehmer selbst zahlen. Ausnahmen sind Rabatte, Muster, Gegenstände von fachlichem Nutzen, zum Beispiel Patiententagebücher, oder Mahlzeiten bis zu einem Wert von 150 Franken. «Die Initiative kommt aus der Industrie», sagte Borer. "Wir wollen eine gesetzliche Regel vermeiden.» Eine hohe Transparenz sei das Ziel, was aber nicht unbedingt zu einem besseren Ruf führe.

$\mathrm{Zu}$ einem schlechten Ruf könnte auch die zunehmende Ökonomisierung in der Medizin führen. Mit zunehmendem Können, etwa durch Katheter-Eingriffe, ergeben sich steigende Gewinnmöglichkeiten - die Medizin werde mehr und mehr zum Business, erzählte Thomas Lüscher. So gibt es in der Schweiz 74 Schrittmacher-Implantationszentren, in denen zwischen zwölf und 350 Implantationen pro Jahr durchgeführt werden. "350 ist eine gute Menge», sagt Lüscher. "Zwölf ist viel zu wenig - die Ärzte haben dann kaum Erfahrung und das Risiko für Komplikationen steigt. Wenn Leistungsaufträge ohne Mindestzahlen oder ohne Zertifizierung vergeben werden, ist das ein Riesenproblem.» Doch der Arzt steht unter einem hohen Druck: Er muss die Kosten für die Gesellschaft gegen den Nutzen für den Einzelpatienten abwägen und schauen, dass das Budget stimmt. In Deutschland, erzählte Lüscher, verlange die Klinik-Kette Fresenius von ihren Krankenhäusern 15 Prozent Gewinn. Ärzte und nichtärztliche Arbeitnehmer klagen über einen hohen Druck. «Wie viel Marketing verträgt denn die Medizin?» fragte Lüscher provokant und machte Lust, seinen soeben erschienenen Artikel zu diesem Thema zu lesen (2). Die Waage müsse im Gleichgewicht sein, forderte Lüscher: Auf der einen Seite Wettbewerb mit Marketing, Kostenoptimierung, Effizienz und Service, auf der anderen Seite Regulierung durch Weiterbildungen der Mitarbeiter, Zertifizierungen der Kliniken, Mindestzahlen, Outcomes-Forschung und den PKK. "Allein die Dosis macht, dass ein Ding kein Gift ist", zitierte Lüscher Paracelsus. Die Dosis von Informationen und Diskussionen über Medizin, politische und industrielle Entscheidungen war auf der CARTAVeranstaltung jedenfalls genau richtig.

\section{Referenzen}

1 www.obsan.admin.ch/bfs/obsan/de/index/o2/o2/o1. Document.174136.pdf

2 Duengen HD, Trippel TD, Fritschka M, Tahirovic E, Wilck N, Busjahn A, et al. Autoantibodies against the beta1 adrenoreceptor detected by ELISA in chronic heart failure patients - from the CIBIS-ELD Trial. Cardiovascular Medicine. 2014;17: Suppl 24:3-7. 